\title{
ON THE ANGULAR DEPENDENCE OF BOMBARDMENT INDUCED LIGHT EMISSION
}

\author{
D. Ghose \\ Saha Institute of Nuclear Physics \\ Sector-1, Block-AF, Bidhan Nagar, Calcutta 700 064, India \\ (Received September 16, 1992; revised version January 26, 1993)
}

The angle for maximum photon yield is compared with that predicted for total sputtering yield. It has been pointed out that the maximum photon yield angle and the sputter yield angle do not occur at the same position. The results are discussed.

PACS numbers: 79.20.Nc

Ion bombardment of solid targets leads to the emission of light either from sputtered or backscattered particles or from the solid materials [1]. Since a fraction of the sputtered particles leaves in some excited state and participates in the photon emission, it is of interest to compare the dependence of the sputtering yield on various parameters with the analogous dependence of light emission. Though the mechanisms of sputtered-atom excitation are not well understood, it is often assumed that the energy distributions of sputtered atoms in the excited state and in the ground state are identical. This implies that the light intensity from the sputtered atoms should be proportional to the total sputtering yield as it is evident from the energy dependence of light intensity measurements [2]. However, some recent experiments $[3,4]$ show that such a correspondence may not be always valid. In this communication, the angular dependence of excited atom yields is compared to that of the total sputtering yield in order to test further the relation of atomic excitation with the sputtering process.

Qualitatively, a typical angular dependence of photon yield curve is found to be similar to that of the neutral sputtering yield, i.e. the yield first rises monotonically with $\theta$, the angle of ion incidence with respect to the surface normal, passes through a maximum at $\hat{\theta}$ typically $60^{\circ}-80^{\circ}$ and then decreases sharply as $\theta$ approaches $90^{\circ}$. For not-too-oblique incidence, Sigmund [5] predicted a $(\cos \theta)^{-f}$ dependence rather than the normally expected $(\cos \theta)^{-1}$ trend, where $1<f<2$. Quite recently, Yamamura [6] proposed an empirical formula containing two adjustable parameters for the angular dependence of sputtering yield covering the whole angular range. One of the parameters corresponds to Sigmund's $f$, while the other is related to $\hat{\theta}$. 
From the analogy of ion reflection with channeling at grazing incidence, $\hat{\theta}$ can be estimated from Lindhard's planar channeling calculations [7]. Ghose [8] proposed the following expression for the angle of incidence at the maximum sputtering yield:

$$
\hat{\theta}=90-178.2\left[\frac{n Z_{1} Z_{2}}{\left(Z_{1}^{2 / 3}+Z_{2}^{2 / 3}\right) E}\right]^{1 / 3},
$$

where $\hat{\theta}$ is in degrees, $Z_{1}$ and $Z_{2}$ are the atomic numbers of bombarding ion and target atom, respectively, $E$ is the ion energy in $\mathrm{eV}$ and $n$ is the number of target atoms per $(\AA)^{3}$. Equation (1) prediets $\hat{\theta}$ values with reasonable accuracy at both low and high keV energies and also for different projectile-target systems [9].

TABLE

Comparison of peak angular positions $\hat{\theta}$ for photon yield and sputtering yield; experiment and theory.

\begin{tabular}{c|c|r|r|r|r|c|c|c}
\hline \hline Target & Projectile & $\begin{array}{c}E \\
(\mathrm{keV})\end{array}$ & $\begin{array}{c}\hat{\theta}^{a} \text { exp. } \\
\left({ }^{\circ}\right)\end{array}$ & Ref. & $\begin{array}{c}\hat{\theta}^{b} \text { exp. } \\
\left(^{\circ}\right)\end{array}$ & Ref. & $\begin{array}{c}\hat{\theta}^{c} \text { theor. } \\
\left({ }^{\circ}\right)\end{array}$ & $\begin{array}{c}\hat{\theta}^{d} \text { theor. } \\
\left({ }^{\circ}\right)\end{array}$ \\
\hline $\mathrm{Al}$ & $\mathrm{Ar}^{+}$ & 40 & 75 & {$[10]$} & & & 84.5 & 80.7 \\
& & 300 & $>80$ & {$[2]$} & & & 87.2 & 84.1 \\
$\mathrm{Ni}$ & $\mathrm{Ar}^{+}$ & 40 & 80 & {$[10]$} & & & 82.6 & 79.2 \\
$\mathrm{Cu}$ & $\mathrm{Ar}^{+}$ & 27 & 76.4 & {$[11]$} & 80 & {$[13]$} & 81.7 & 78.4 \\
& & 40 & 75 & {$[10]$} & $81^{*}$ & {$[14]$} & 82.7 & 79.4 \\
$\mathrm{Zn}$ & \multirow{2}{*}{$\mathrm{He}^{+}$} & 8 & 55 & {$[12]$} & & & 83.7 & 77.6 \\
\hline
\end{tabular}

a Exp. data for photon yield.

${ }^{b}$ Exp. data for sputt. yield.

$c$ Theor. values of Ghose.

${ }^{d}$ Theor. values of Yamamura.

* Data extracted from measured cone apex angle.

The theoretical values of sputter peak angles are compared with the experimentally obtained photon yield maximum angles and they are displayed in Table. The values of $\hat{\theta}$ obtained following the prescriptions of Yamamura [6] are also included. Table shows that, except for $8 \mathrm{keV} \mathrm{He}^{+} \rightarrow \mathrm{Zn}$ combination, the agreement between the calculated and measured $\hat{\theta}$ values is quite satisfactory. It is interesting to note that the photon yield peak generally occurs before the sputtering yield maximum, which is also evident from the only available experimental data for both $\hat{\theta}$ at the same energy for $\mathrm{Cu}$ target (Table). This is probably the reason for a large discrepancy between $\hat{\theta}$ (exp. photon yield) and $\hat{\theta}$ (theor.) values for some cases.

When the target surface is tilted, the enhancement of photon yield is, mainly, the result of two effects: the increase in total sputtering yield and in the number of high velocity sputtered atoms. Because of strong excitation of some selected levels, Larsen and Veje [15] proposed an additional mechanism for the increase 
in light emission. At large tilt angle, the recoil target atoms experience violent binary collisions before leaving the target surface. This may cause atomic excitation through molecular-orbital electron-promotion processes. However, the latter process cannot be accounted for the change in position of the photon yield peak with respect to the sputter yield peak.

A plausible explanation for the difference in positions of the sputter yield peak and photon yield peak can be given on the basis of the works of Gabla and Suchaniska [4]. They concluded that the focused energy packets transmitted in a series of head-on collisions do not contribute to the excitation of the end atoms. It is well known that for perpendicular ion incidence particle ejection occurs symmetrically to the target normal. But for oblique ion incidence the atoms are preferentially sputtered in the forward direction. At grazing ion incidence where the sputtering yield is maximum, the collision cascade develops at the surface and the maximum ejection direction is shifted from the vicinity of the target normal into the region of specular reflection. In this situation, most of the recoiled atoms are involved in the anisotropic emission and one may draw the analogy with a monocrystalline target that the ejection of atoms is the result from a sequence of collisions with focusing of the momenta in the forward direction. Thus the absence of random atomic collisions might be the reason for the reduction of photon emission yield at the sputter yield peak position. In passing, it is worth mentioning the photon emission studies under channeling conditions of van der Weg et al. [16] and Martin and MacDonald [17]. Pronounced intensity minimum in the optical radiation is observed, when the incoming beam is aligned with a major crystallographic direction of the target crystal. A channeling spectrum is characterized by a dip and shoulders. While the former is due to channeled particles which cannot come close to the atomic positions, the latter is caused by quasi-channeled particles hitting the exact direction of the string of lattice atoms. As the correlated sequences of collisions propagate along close packed lines of atoms in the crystal, the final collision with the topmost atom results in sputtering of that atom. If this atom is not excited then the angular widths of the channeling minima will be wider. Indeed this is experimentally found when compared with Lindhard's estimate [7], thus supporting further the evidences of Gabła and Suchaniska [4] as well as the present results.

In summary, though the maximum photon yield angle can be predicted reasonably well by the expressions developed for that of sputtering yield, the data show that the two maxima do not appear at the same position. Since at the maximum yield angle the collision cascade develops at the surface and the atoms are predominantly sputtered in the forward direction, one may think some sort of focusing of momenta taking place in that direction. Experimental evidences [4] show that the atoms originating from focused collision sequences do not emit photons. Although this explains partly the discrepancy between the experimental and theoretical $\hat{\theta}$ values, other phenomena such as the development of sputtering induced surface topography may play a role for the difference. One may, therefore, conclude that the photon yield is not always linearly proportional to the sputtering yield, at least near the angles where the emission of sputtered atoms is maximum. 


\section{References}

[1] E.W. Thomas, Prog. Surf. Sci. 10, 383 (1980).

[2] S. Reinke, R. Hippler, Nucl. Instrum. Methods Phys. Res. B 67, 620 (1992).

[3] I.N. Evdokimov, I.M. Fayazov, E.S. Mashkova, V.A. Molchanov, V.A. Snisar, Radiat. Eff. Def. Solid 112, 221 (1990).

[4] L. Gabła, M. Suchańska, J. Phys., Condens. Matter 3, 6159 (1991).

[5] P. Sigmund, Phys. Rev. 184, 383 (1969).

[6] Y. Yamamura, Radiat. Eff. 80, 57 (1984); Y. Yamamura, Y. Itikawa, N. Itoh, Inslitute of Plasma Physics Report, IPPJ - AM - 26, Nagoya University, Nagoya 1983.

[7] J. Lindhard, Mat.-Fys. Medd. K. Dan. Vidensk. Selsk. 34, no. 14 (1965).

[8] D. Ghose, Jpn. J. Appl. Phys. 18, 1847 (1979).

[9] D. Ghose, S.B. Karmohapatro, in: Advances in Electronics and Electron Physics, Ed. P. Hawkes, Vol. 79, Academic Press, New York 1990, p. 73.

[10] J.J. Jimenez-Rodriguez, D.S. Karpuzov, D.G. Armour, Surf. Sci. 136, 155 (1984).

[11] A.M. Borisov, Radiat. Eff. 97, 85 (1986).

[12] L. Gabła, R. Pedrys, M. Szymoński, Acta Phys. Pol. A 51, 311 (1977).

[13] I.N. Evdokimov, V.A. Molchanov, Sov. Phys.-Solid State 9, 1967 (1968).

[14] L. Tanović, J.L. Whitton, S. Kofod, in: Proc. Conf. Physics of Ionized Gases, SPIG 78, Ed. R.K. Janev, Institute of Physics, Beograd, Yugoslavia 1978, p. 171.

[15] P. Larsen, E. Veje, Phys. Rev. B 28, 5011 (1983).

[16] W.F. van der Weg, N.H. Tolk, C.W. White, J.M. Kraus, Nucl. Instrum. Methods 132, 405 (1976).

[17] P.J. Martin, R.J. MacDonald, Radiat. Eff. 32, 177 (1977). 